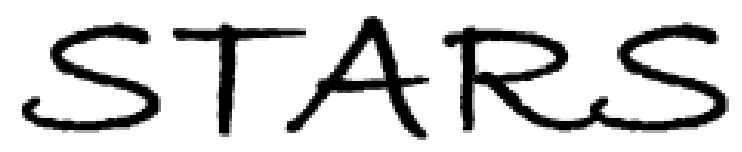

University of Central Florida

STARS

Faculty Bibliography 2010s

Faculty Bibliography

$1-1-2010$

\title{
Triple-junction contribution to diffusion in nanocrystalline Si
}

\author{
A. Portavoce \\ L. Chow \\ University of Central Florida \\ J. Bernardini
}

Find similar works at: https://stars.library.ucf.edu/facultybib2010 University of Central Florida Libraries http://library.ucf.edu

This Article is brought to you for free and open access by the Faculty Bibliography at STARS. It has been accepted for inclusion in Faculty Bibliography 2010 s by an authorized administrator of STARS. For more information, please contact STARS@ucf.edu.

\section{Recommended Citation}

Portavoce, A.; Chow, L.; and Bernardini, J., "Triple-junction contribution to diffusion in nanocrystalline Si" (2010). Faculty Bibliography 2010s. 651.

https://stars.library.ucf.edu/facultybib2010/651

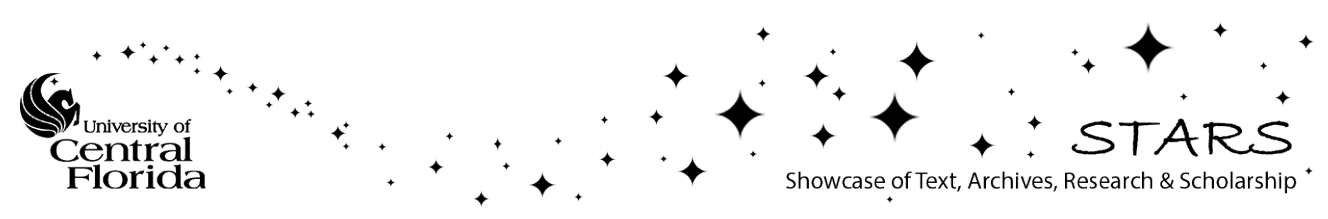




\section{Triple-junction contribution to diffusion in nanocrystalline Si}

Cite as: Appl. Phys. Lett. 96, 214102 (2010); https://doi.org/10.1063/1.3435476

Submitted: 11 March 2010 . Accepted: 30 April 2010 . Published Online: 24 May 2010

A. Portavoce, L. Chow, and J. Bernardini

芈

\section{ARTICLES YOU MAY BE INTERESTED IN}

Grain boundary and triple junction diffusion in nanocrystalline copper

Journal of Applied Physics 116, 093514 (2014); https://doi.org/10.1063/1.4893960

Geometric considerations for diffusion in polycrystalline solids

Journal of Applied Physics 101, 063524 (2007); https://doi.org/10.1063/1.2711820

\section{Applied Physics Reviews} Now accepting original research 


\title{
Triple-junction contribution to diffusion in nanocrystalline $\mathbf{S i}$
}

\author{
A. Portavoce, ${ }^{1, a)}$ L. Chow, ${ }^{2}$ and J. Bernardini ${ }^{1}$ \\ ${ }^{1}$ IM2NP, Faculté des Sciences de Saint-Jérôme, CNRS, Case 142, 13397 Marseille, France \\ ${ }^{2}$ Department of Physics, University of Central Florida, Orlando, Florida 32816, USA
}

(Received 11 March 2010; accepted 30 April 2010; published online 24 May 2010)

\begin{abstract}
The influence of triple-junctions on experimental Ge diffusion profiles $\left(850-1000{ }^{\circ} \mathrm{C}\right)$ in nanocrystalline $\mathrm{Si}$ is investigated using three-dimensional finite element simulations. We found that triple-junction diffusion is not negligible in nanocrystalline $\mathrm{Si}$ made of $40 \mathrm{~nm}$ wide grains. Ge triple-junction diffusion coefficient follows the Arrhenius law 5.72 $\times 10^{4} \exp (-3.24 \mathrm{eV} / \mathrm{kT}) \mathrm{cm}^{2} \mathrm{~s}^{-1}$. It is approximately $4.7 \times 10^{2}$ times higher than grain boundary diffusion coefficient, even though diffusion in triple-junction and in grain boundary exhibits similar activation energy. (C) 2010 American Institute of Physics. [doi:10.1063/1.3435476]
\end{abstract}

Nanocrystalline (nc) materials are of great interest for many industrial applications. For example, nanocrystalline $\mathrm{Si}$ (nc-Si) can be used in telecommunication applications ${ }^{1}$ and in photodetector fabrication. ${ }^{2}$ Hydrogenated $\mathrm{nc}-\mathrm{Si}$ is a good candidate for solar cell fabrication: ${ }^{3-5}$ (i) it exhibits a low production cost, (ii) it can be used for all-Si tandem solar cell applications, ${ }^{3}$ since nc-Si band gap can be controlled from 1 to $3 \mathrm{eV}$ by controlling nanograin size, ${ }^{5}$ (iii) quantum confinement and quantum effects can improve photocarrier transport and cell efficiency, ${ }^{5}$ and can allow multiple carrier generation. ${ }^{4}$ Nc-material production generally involves atomic diffusion during their processes. For example, applications such as photoluminescence, electroluminescence, ${ }^{6}$ and solar cells ${ }^{7}$ require Si nanocrystals or nc-Si layers to be doped with one or several impurities. Impurity diffusion studies in nc-materials, and in particular in nc-Si is thus very important, since mechanisms as self-purification, ${ }^{8}$ and structural properties of nc-materials can modify atomic transport properties, and complicate the doping processes. Triplejunctions (TJs), which correspond to the intersection of grain boundaries (GBs) in polycrystals, should play an important role upon atomic diffusion in nc-layers, as their density increases when grain size decreases. The properties of these one-dimensional defects are still not very well known. Theoretical considerations agree that TJs should be treated as independent defects, different than interfaces and GBs. ${ }^{9}$ Recent molecular dynamic (MD) simulations by Frolov and Mishin $^{10}$ showed that in $\mathrm{Cu}$ TJs exhibit a higher positive excess energy than GBs. Other MD simulations ${ }^{11}$ showed that in $\mathrm{Si}$, TJs are different defects than GBs with positive excess energy, and are source of residual stress. Positron experiments on nc-Fe (Ref. 12) confirmed that TJs and GBs are different: free volumes at interfaces like GBs correspond to single vacancies, while interface intersections as TJs exhibit microvoids that correspond to vacancy agglomeration. The influence of TJs on impurity diffusion in nc-materials depends upon impurity mobility in TJs and TJs volume proportion, i.e., the TJ contribution is not negligible if $f_{t j} D_{t j}$ $\geq f_{g b} D_{g b} \geq f_{g} D_{g}$, with $f_{t j}, f_{g b}$, and $f_{g}$ the respective volume fraction of TJs, GBs, and grains, and $D_{t j}, D_{g b}$, and $D_{g}$ the diffusion coefficients in TJs, GBs, and grains, respectively.

\footnotetext{
a) Author to whom correspondence should be addressed. Electronic mail: alain.portavoce@im2np.fr.
}

TJ effect is expected to be significant at low temperature, at which $D_{t j} \gg D_{g b}$ and $D_{g}$, and for grain size lower than $10 \mathrm{~nm}$, since in this case $f_{g} \leq 0.8, f_{g b} \geq 0.2$ and $f_{t j} \geq 0.02 .{ }^{13}$ However, Chen and Schuh ${ }^{14}$ suggested that TJs can influence diffusion in nc-materials made of $100 \mathrm{~nm}$ wide grains, for temperatures lower than $0.8 \times T_{m}$, with $T_{m}$ the matrix melting temperature. Experimentally, nc-materials exhibit diffusivities of several orders of magnitude larger than in microcrystalline (mc) materials. However, due to the difficulty to separate the TJ contribution from the GB contribution in diffusion profiles, it is not clear if these results can be attributed to a higher diffusivity in nano-GBs compared to microGBs, or to a higher diffusivity in TJs compared to GBs. Diffusion coefficient measurements performed in polycrystalline materials using models that consider TJs are quite rare, ${ }^{15}$ the Fisher model ${ }^{16}$ being generally used to study diffusion in polycrystals. For example, $\mathrm{Cu}$ GB diffusion coefficient measured at $0.23-0.27 \times T_{m}$ in nc-Ni (grain sizes 100 $300 \mathrm{~nm}$ ) was found to be $10^{5}$ times faster than in mc-Ni. ${ }^{17} \mathrm{Zn}$ diffusion in polycrystalline $\mathrm{Al}$ was clearly shown to be faster in TJs than in GBs, ${ }^{18}$ with $D_{t j} / D_{g b} \sim 10^{3}$ at $0.6 \times T_{m}$. Furthermore, Chen and Schuh ${ }^{19}$ showed that using a diffusion model taking into account TJs, experimental studies on metals performed at $\sim 0.2-0.3 \times T_{m}$ give $10^{3} \leq D_{t j} / D_{g b} \leq 10^{8}$.

Despite the high potential of nc-semiconductors for industrial applications, studies treating of TJ influence upon atomic transport in these materials are rare. In a previous work, ${ }^{20}$ we measured and compared Ge diffusivity in grains (lattice diffusion) and GBs in microcrystalline and nanocrystalline $\mathrm{Si}\left(40 \mathrm{~nm}\right.$ wide grains) at $850-1000{ }^{\circ} \mathrm{C}$, using Fisher's model and two-dimensional (2D model) finite element simulations (FES). Ge diffusion in $\mathrm{Si}$ is ideal to study diffusion in nc-semiconductors since: (i) $\mathrm{Si}(\mathrm{Ge}$ ) layers can be produced with microelectronics purity level and ideal density (no porosity), (ii) nc-Si layers are produced without compaction or severe plastic deformations that can add complexity, (iii) $\mathrm{Ge}$ is a fully soluble substitutional impurity in $\mathrm{Si}$, (iv) Ge lattice diffusion uses the same vacancy mechanism in our experimental conditions, and (v) Ge segregation in Si GBs has not been observed. The Ge diffusion coefficients in nc-Si (nc- $D_{g}$ and nc- $D_{g b}$ ) were found about one order of magnitude faster than in mc-Si $\left(\mathrm{mc}-D_{g}\right.$ and $\left.\mathrm{mc}-D_{g b}\right)$. The activation energy $\left(E_{a}\right)$ was found about $1 \mathrm{eV}$ lower in nanograins $\left(\mathrm{nc}-E_{g}\right)$ than in micrograins $\left(\mathrm{mc}-E_{g}\right)$, while similar $E_{a}$ was 


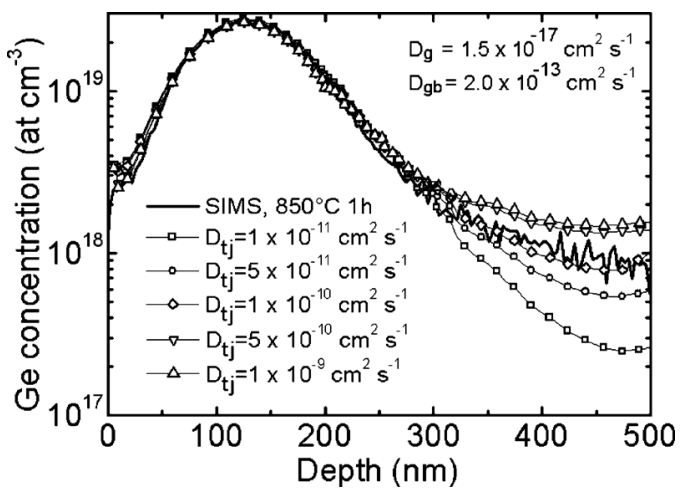

FIG. 1. SIMS profile after annealing at $850{ }^{\circ} \mathrm{C}$ for $1 \mathrm{~h}$, and simulated profiles with $D_{g}=1.5 \times 10^{-17} \mathrm{~cm}^{2} \mathrm{~s}^{-1}, D_{g b}=2 \times 10^{-13} \mathrm{~cm}^{2} \mathrm{~s}^{-1}$, and $10^{-11}$ $\leq D_{t j} \leq 10^{-9} \mathrm{~cm}^{2} \mathrm{~s}^{-1}$.

found in nano-(nc- $\left.E_{g b}\right)$ and micro-GBs $\left(\mathrm{mc}-E_{g b}\right)$.

The present study investigates TJ influence on Ge diffusion in nc-Si in the $0.67-0.76 \times T_{m}$ temperature range. We used the same experimental Ge diffusion profiles as presented in Ref. 20. The fitting process was the same as described in Ref. 20, a cubic geometry similar to Fisher's model was used to extract diffusion coefficients, except that FES using a three-dimensional (3D) model including TJs was employed. In this geometry, the three diffusion coefficients $D_{g}, D_{g b}$, and $D_{t j}$ can be extracted but not independently. ${ }^{20,2}$ Furthermore, the contribution fractions of grains, GBs and TJs are different in the $2 \mathrm{D}$ and $3 \mathrm{D}$ geometries, with $f_{g}=0.98765, f_{g b}=0.01235$, and $f_{t j}=0.0$ for the $2 \mathrm{D}$ model, and $f_{g}=0.97546, f_{g b}=0.02439$, and $f_{t j}=0.00015$ for the 3D model. Nevertheless, for same $D_{g}$ and $D_{g b}$ (with $D_{g b}=D_{t j}$ for the 3D model) the simulated profiles of the 2D and 3D models were found to be very similar. For $40 \mathrm{~nm}$ grains, differences less than $20 \%$ were found in diffusion coefficients between the 2D and 3D models. Figure 1 presents the Ge experimental secondary ion mass spectrometry (SIMS) profile measured in the nc-Si layer after annealing at $850^{\circ} \mathrm{C}$ for one hour, as well as simulated profiles obtained for the same annealing conditions with same $D_{g}=1.5$ $\times 10^{-17}$ and $D_{g b}=2 \times 10^{-13} \mathrm{~cm}^{2} \mathrm{~s}^{-1}$, but for various $D_{t j}$ values, from $10^{-11}$ to $10^{-9} \mathrm{~cm}^{2} \mathrm{~s}^{-1} . D_{g}$ and $D_{g b}$ correspond to the nanograin diffusion coefficient, and to the micro-GB diffusion coefficient measured at this temperature in Ref. 20, respectively. The variation in $D_{t j}$ corresponds to a ratio $D_{t j} / D_{g b}$ comprise between $5 \times 10^{1}$ and $5 \times 10^{3}$, which is realistic considering previous works. ${ }^{18,19}$ This figure clearly shows that Ge diffusivity in TJs cannot be neglected in nc-Si made of $40 \mathrm{~nm}$ wide grains, since the final 1D Ge profile is highly dependent upon the value of $D_{t j}$. Furthermore, the Ge experimental profile can be fitted using the same GB diffusion coefficient as measured in $\mathrm{mc}-\mathrm{Si}$ and considering a TJ diffusion coefficient of about 1 $\times 10^{-10} \mathrm{~cm}^{2} \mathrm{~s}^{-1}$. As we showed in Ref. 20, $D_{g}$ and $D_{g b}$ can be determined simultaneously from FES if the value of $D_{t j}$ is known. In order to understand the effect of TJ diffusivity on the coefficients that we measured in nanograins and nanoGBs using the 2D Fisher geometry, we fixed the value of $D_{t j}$ to $10^{-9} \mathrm{~cm}^{2} \mathrm{~s}^{-1}$, and extracted the values of $D_{g}$ and $D_{g b}$ by fitting the experimental $\mathrm{Ge}$ diffusion profiles. ${ }^{2{ }^{g b}} D_{t j}$ $=10^{-9} \mathrm{~cm}^{2} \mathrm{~s}^{-1}$ is at least two orders of magnitude higher than the GB diffusion coefficient we measured in $\mathrm{mc}-\mathrm{Si},{ }^{20}$ while it is at least three orders of magnitude lower than $\mathrm{Si}$

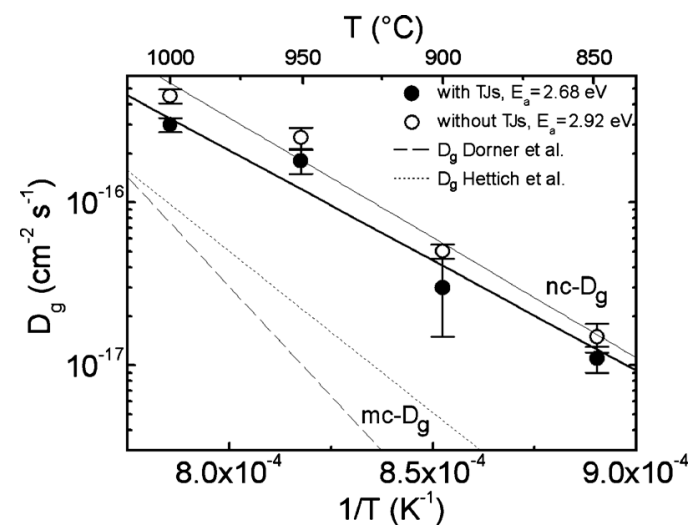

FIG. 2. Nc- $D_{g}$ from $2 \mathrm{D}$ simulations without TJs (open symbols) and from $3 \mathrm{D}$ simulations with TJs (solid symbols) with $D_{t j}=1 \times 10^{-9} \mathrm{~cm}^{2} \mathrm{~s}^{-1}$. Ge lattice diffusion coefficients from Dorner et al. (dashed line) and Hettich et al. (dotted line) are also presented.

surface self-diffusion ${ }^{22}$ in our temperature range. Figure 2 presents the temperature dependence of the diffusion coefficients in nanograins (nc- $D_{g}$ ) measured using the 2D geometry without TJs (open symbols) (Ref. 20) and using the 3D geometry with TJs (solid symbols), as well as the diffusion coefficients measured in monocrystalline $\mathrm{Si}\left(\mathrm{mc}-D_{g}\right)$ by Dorner et $a l^{23}$ (dashed line) and Hettich et al. ${ }^{24}$ (dotted line). The coefficients measured using the two different geometries are very similar, with similar activation energies. This is due to the very small surface area of grain /TJ interfaces, which leads to negligible direct atom exchanges between grains and TJs. The same conclusion as made in Ref. 20 can be given with the 3D geometry: nc- $D_{g}$ is one order of magnitude higher than mc- $D_{g}$, and nc- $E_{g}$ is at least $1 \mathrm{eV}$ smaller than mc- $E_{g}$. Figure 3 presents the temperature dependence of nc- $D_{g b}$ measured using the two different geometries without (open symbols) and with TJs (solid symbols), as well as mc- $D_{g b}$ using the lattice diffusion coefficients of Dorner et al. (dotted line) or Hettich et al. (dashed line). The activation energies in nano-GBs measured using the 2D (nc- $E_{g b}$ $\sim 3.54 \mathrm{eV})$ and the 3D (nc- $\left.E_{g b} \sim 3.80 \mathrm{eV}\right)$ geometries are comparable. However, the $3 \mathrm{D}$ nc- $D_{g b}$ is found about one order of magnitude lower than the $2 \mathrm{D}$ nc- $D_{g b}$. It can be noticed that considering a constant $D_{t j}=10^{-9} \mathrm{~cm}^{2} \mathrm{~s}^{-1}$, the $3 \mathrm{D}$ nc- $D_{g b}$ is found to be similar to the mc- $D_{g b}$ (using $D_{g}$ of

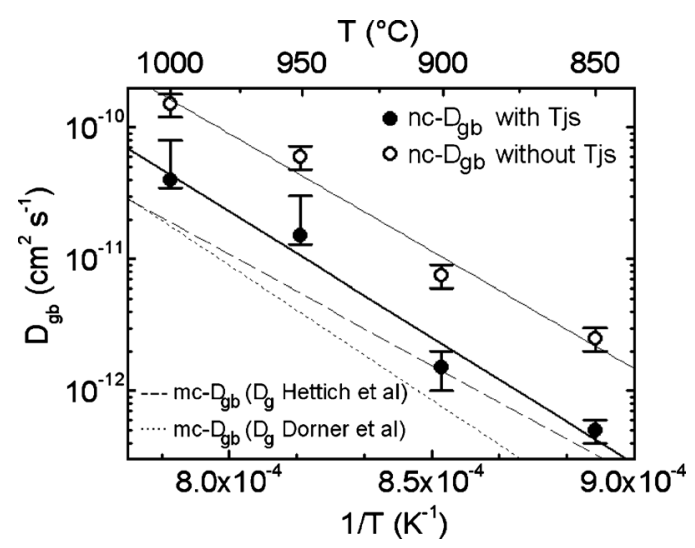

FIG. 3. Nc- $D_{g b}$ from 2D simulations without TJs (open symbols) and from 3D simulations with TJs (solid symbols) with $D_{t j}=1 \times 10^{-9} \mathrm{~cm}^{2} \mathrm{~s}^{-1}$. Ge GB diffusion coefficients measured in mc-Si using the Ge lattice diffusion coefficient either from Dorner et al. (dotted line) or Hettich et al. (dashed line) are also presented. 


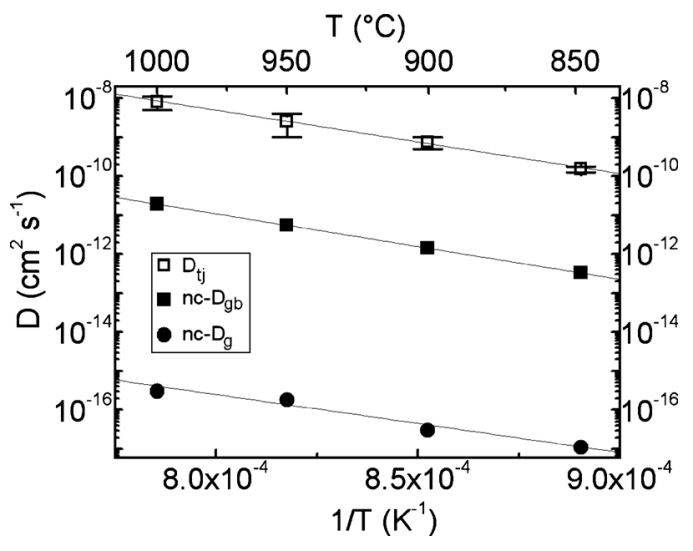

FIG. 4. $D_{t j}$ (open squares) and nc- $D_{g}$ (solid circles) measured in $\mathrm{nc}-\mathrm{Si}$ considering that nc- $D_{g b}$ is the same as mc- $D_{g b}$ (solid squares).

Hettich et al.), for the two lower temperatures (900 and $850^{\circ} \mathrm{C}$ ). This observation suggests that TJ diffusion coefficients at 1000 and $950{ }^{\circ} \mathrm{C}$ are higher than $10^{-9} \mathrm{~cm}^{2} \mathrm{~s}^{-1}$. Considering an average TJ effect (constant $D_{t j}$ ) upon Ge diffusion in nc-Si, nano-GB diffusion coefficients are found to be close to micro-GB diffusion coefficients. Combining the results obtained on Ge diffusion in nc-Si without taking into account TJs (Ref. 20) and considering TJs, it can be conclude that (i) nc- $D_{g}$ is faster than mc- $D_{g}$ : the nanosize effect increases the vacancy concentration in nanograins compared to bulk, due to the influence of the grain surfaces ${ }^{20}$ (Beckman and Chelikowsky ${ }^{25}$ have shown that reducing Si nanocrystal size can decrease the formation and the migration energies of Si vacancies), (ii) nc- $D_{g b}$ and mc- $D_{g b}$ are the same: the nanosize effect does not drastically change the nature of GBs ${ }^{20}$ in agreement with the work of Caro and Van Swygenhoven ${ }^{26}$ showing that nano-GBs and micro-GBs exhibit similar structures, size, energies and disorder, and (iii) Tjs have an effect on $\mathrm{Ge}$ diffusion even for $40 \mathrm{~nm}$ wide $\mathrm{Si}$ grains. Consequently, the 2D model cannot be used to measure grain boundary diffusion in nanocrystalline layers. The Ge TJ diffusivity in nc-Si can be measured in our experiments using 3D FES, considering $D_{g}$ to be the same as the nc- $D_{g}$ measured in Ref. 20, and $D_{g b}$ to be the same as the mc- $D_{g b}$ measured in Ref. 20. Figure 4 presents the Ge TJ diffusion coefficients measured in nc-Si (open symbols), as well as the nc- $D_{g}$ (solid circles) and the mc- $D_{g b}$ (solid squares) from Ref. 20. The Ge TJ diffusion coefficient is found to be $D_{j t}=5.72 \times 10^{4} \exp (-3.24 \mathrm{eV} / \mathrm{kT}) \mathrm{cm}^{2} \mathrm{~s}^{-1}$, with an average $D_{t j} / D_{g b} \sim 4.7 \times 10^{2} . D_{t j}$ and $D_{g b}$ are found to have similar activation energy, meaning that the difference between the two coefficients is mainly due to different Arrhenius prefactors $\left(D_{0}\right)$. This is in agreement with experimental ${ }^{12}$ and theoretical ${ }^{10}$ works showing that the principal difference between GBs and TJs is a higher disorder degree in TJs. $D_{0}$ contains, among several factors, the entropic component that is proportional to disorder, a higher $D_{0}$ corresponding to a higher degree of disorder. Similar to our observations, Frolov and Mishin ${ }^{10}$ predicted a faster Cu self-diffusivity in TJs than in GBs mainly due to a larger TJ Arrhenius prefactor than the
GB one $\left(\mathrm{TJ}-E_{a}=0.47 \mathrm{eV}\right.$ and $\left.\Sigma 5 \mathrm{~GB}-E_{a}=0.48 \mathrm{eV}\right)$. However, Frolov and Mishin ${ }^{10}$ predicted a TJ $D_{0}$ only two times higher than the GB $D_{0}$. This difference with experimental results may be due to an entropy increase related to the additional chemical disorder involved with impurity diffusion in experiments, instead of self-diffusion in simulations. In addition, Belova and $\mathrm{Much}^{27}$ proposed that the high diffusion rate observed experimentally can be due to multiple-atomjump mechanisms.

In conclusion, Ge diffusion in $\mathrm{nc}-\mathrm{Si}$ has been investigated using 3D FES in the $0.67-0.76 \times T_{m}$ temperature range. TJ diffusion is not negligible for Ge diffusion in nc-Si made of $40 \mathrm{~nm}$ wide grains. Consequently, 2D models cannot be used to extract grain boundary diffusion in nanocrystalline layers. Ge lattice diffusion is found one order of magnitude faster in $\mathrm{Si}$ nanograins compared to monocrystalline $\mathrm{Si}$. Ge diffusion is identical in nano-GBs and in micro-GBs, while Ge diffusion is faster in Tjs $\left(\times 4.7 \times 10^{2}\right)$ than in GBs. This work shows that Ge enhanced diffusion in nc-Si results from two contributions: (i) faster lattice diffusion in nanograins and (ii) fast diffusion in TJs.

${ }^{1}$ R. Zhang, X. Y. Chen, and W. Z. Shen, J. Appl. Phys. 105, 033718 (2009). ${ }^{2}$ S.-K. Kim, B.-H. Kim, C.-H. Cho, and S.-J. Park, Appl. Phys. Lett. 94, 183106 (2009).

${ }^{3}$ J. Yang, B. Yan, and S. Guha, Thin Solid Films 487, 162 (2005).

${ }^{4}$ M. C. Beard, K. P. Knutsen, P. Yu, J. M. Luther, Q. Song, W. K. Metzger, R. J. Ellingson, and A. J. Nozik, Nano Lett. 7, 2506 (2007).

${ }^{5}$ R. Zhang, H. Wu, X. Chen, and W. Shen, Appl. Phys. Lett. 94, 242105 (2009).

${ }^{6}$ K. Murakami, R. Shirakawa, M. Tsujimura, N. Uchida, N. Fukata, and S.-I. Hishita, J. Appl. Phys. 105, 054307 (2009).

${ }^{7}$ K. Sato, N. Fukata, and K. Hirakuri, Appl. Phys. Lett. 94, 161902 (2009).

${ }^{8}$ G. M. Dalpian and J. R. Chelikowsky, Phys. Rev. Lett. 96, 226802 (2006).

${ }^{9}$ A. H. King, Interface Sci. 7, 251 (1999).

${ }^{10}$ T. Frolov and Y. Mishin, Phys. Rev. B 79, 174110 (2009).

${ }^{11}$ S. Costantini, P. Alippi, L. Colombo, and F. Cleri, Phys. Rev. B 63, 045302 (2000).

${ }^{12}$ H.-E. Schaefer, R. Würschum, R. Birringer, and H. Gleiter, Phys. Rev. B 38, 9545 (1988).

${ }^{13}$ I. A. Ovid'ko and A. G. Sheinerman, Rev. Adv. Mater. Sci. 6, 41 (2004)

${ }^{14}$ Y. Chen and C. A. Schuh, J. Appl. Phys. 101, 063524 (2007).

${ }^{15}$ L. M. Klinger, L. A. Levin, and A. L. Peletin, Defect Diffus. Forum 143-147, 1523 (1997).

${ }^{16}$ J. C. Fisher, J. Appl. Phys. 22, 74 (1951).

${ }^{17}$ Yu. R. Kolobov, G. P. Grabovetskaya, I. V. Ratochka, and K. V. Ivanov, Nanostruct. Mater. 12, 1127 (1999).

${ }^{18}$ B. Bokstein, V. Ivanov, O. Oreshina, A. Peteline, and S. Peteline, Mater. Sci. Eng., A 302, 151 (2001).

${ }^{19}$ Y. Chen and C. A. Schuh, Scr. Mater. 57, 253 (2007).

${ }^{20}$ A. Portavoce, G. Chai, L. Chow, and J. Bernardini, J. Appl. Phys. 104, 104910 (2008).

${ }^{21}$ I. Blum, A. Portavoce, D. Mangelinck, R. Daineche, K. Hoummada, J. L. Lábár, V. Carron, and C. Perrin, J. Appl. Phys. 104, 114312 (2008).

${ }^{22}$ Y. W. Mo, J. Kleiner, M. B. Webb, and M. G. Lagally, Phys. Rev. Lett. 66, 1998 (1991).

${ }^{23}$ P. Dorner, W. Gust, B. Predel, U. Roll, A. Lodding, and H. Odelius, Philos. Mag. A 49, 557 (1984).

${ }^{24}$ G. Hettich, H. Mehrer, and K. Maier, Inst. Phys. Conf. Ser. 46, 500 (1979).

${ }^{25}$ S. P. Beckman and J. R. Chelikowsky, Physica B 401-402, 537 (2007).

${ }^{26}$ A. Caro and H. Van Swygenhoven, Phys. Rev. B 63, 134101 (2001).

${ }^{27}$ I. V. Belova and G. E. Murch, Philos. Mag. A 83, 2399 (2003). 